EU-Emissionshandel für Verkehr und Gebäude

\section{Ein Vorschlag für sozial abgefederte Kohlenstoffpreise}

\author{
Die Rolle der $\mathrm{CO}_{2}$-Bepreisung im Gebäude- und Verkehrssektor \\ in Deutschland haben Lerch und Rudolph in ÖkologischesWirt- \\ schaften 3/21 analysiert. In ihrem Beitrag befürworten sie einen \\ EU-Emissionshandel für eine effizientere Klimapolitik mit \\ sozialem Ausgleich. Sie befürchteten aber, dass diese Chance \\ nicht konsequent genutzt würde. Auf Grundlage des aktuellen \\ Kommissionsvorschlags können neue Ansatzpunkte für den \\ Emissionshandel entwickelt werden. \\ Von Jan Nill
}

M itte Juli hat die Europäische Kommission ein Gesetzespaket vorgeschlagen, um das gesetzlich festgelegte Europäische Klimaziel für 2030 der Verringerung der Nettoemissionen um mindestens $55 \%$ im Vergleich zu 1990 in verantwortungsvoller Weise zu erreichen (EU 2021). Ein zentraler Vorschlag ist die Stärkung und Erweiterung des Europäischen Emissionshandels in kosteneffizienter Weise bei gleichzeitiger Berücksichtigung der Notwendigkeit eines gerechten Übergangs und des Erfordernisses, dass alle Sektoren zu den Klimaschutzbemühungen der EU beitragen (EC 2021a).

Derzeitentfallen $36 \%$ der direkten und indirekten energiebezogenen Treibhausgasemissionen in der EU auf den Gebäudesektor, der über ein großes kosteneffizientes Potenzial zur Emissionsreduktion verfügt. Über die Hälfte dieser Emissionen, die seit $2014 \mathrm{EU}$-weit angestiegen sind, ist bereits durch das bestehende EU-Emissionshandelssystem abgedeckt. Dies gilt vor allem für die Stromversorgung von Gebäuden und den Großteil der Fernwärmeemissionen. Viele Häuser und Wohnungen werden jedoch immer noch mit veralteten Systemen geheizt, die umweltschädliche fossile Brennstoffe wie Kohle und Öl verwenden. Auch der Straßenverkehrssektor verfügt über ein erhebliches kosteneffizientes Reduktionspotenzial. Gegenwär- tig entfällt ein Fünftel der Treibhausgasemissionen in der EU auf den Straßenverkehr, und seine Emissionen sind seit 1990 um mehr als ein Viertel gestiegen.

Die Kommission schlägt vor, ab 2026 Emissionshandel auch für den Gebäudesektor und den Straßenverkehr einzuführen. Dies würde - im Kontext anderer Regulierungsmaßnahmen - für mehr und stärker harmonisierte wirtschaftliche Anreize sorgen, die Emissionen in diesen beiden Sektoren in der EU zu reduzieren, und somit die Gewissheit erhöhen, dass dies auch geschieht. Die Zielvorgabe bis 2030 is kohärent mit den kosteneffizienten Beiträgen der erfassten Sektoren von $43 \%$ Emissionsminderung im Vergleich zu 2005. Die Ausweitung auf den Gebäudesektor und den Straßenverkehr erfordert einen „Upstream“-Ansatz, der auf Einrichtungen wie Brennstoffanbieter, die den regulierten Sektoren vorgelagert sind, ausgerichtet ist. Dies ist auch im deutschen Brennstoffemissionshandelsgesetz der Fall.

Der neue EU-Emissionshandel sollte über ein separates, aber verwandtes System für diese Sektoren erfolgen. Eine etwaige Zusammenführung mit dem bestehenden EU-EHS sollte erst 2031 auf Grundlage der gesammelten Erfahrungen geprüft werden.

Alle Emissionsrechte im neuen Emissionshandel sollen versteigert werden. Einnahmen könnten in diesen Sektoren zur Abfederung der sozialen Folgen eingesetzt und in Maßnahmen investiert werden, die auf die Beschleunigung der Gebäuderenovierungswelle und die Nutzung emissionsfreier Fahrzeuge sowie auf die Entwicklung der erforderlichen Infrastruktur wie zum Beispiel strategisch platzierter intelligenter Tank- und Ladestationen für emissionsfreie Fahrzeuge abzielen. Unterstützungsmaßnahmen zur Förderung der Energieeffizienz in benachteiligten oder einkommensschwachen Haushalten könnten ebenfalls dazu beitragen, exzessive Verteilungseffekte zu vermeiden. Deshalb schlägt die Kommission zugleich einen KlimaSozialfonds in Höhe von 72,2 Milliarden Euro vor, der $25 \%$ der voraussichtlichen Versteigerungseinnahmen entspricht. Gemäß dem Vorschlag steuern die Mitgliedstaaten finanzielle Mittel in gleicher Höhe bei, sodass der Fonds 144,4 Mrd. Euro für einen sozial gerechten Übergang mobilisieren würde (EC 2021b). Ein besonderer Schwerpunkt liegt auf benachteiligten Haushalten, Kleinstunternehmen und Verkehrsnutzer/innen.

\section{Literatur}

EC (2021 a): Proposal for a Directive of the European Parliament and of the Council amending Directive 2003/87/EC. https://ec.europa. eu/transparency/documents-register/ detail?ref=COM(2021) 551\&lang=en

EC (2021 b): Proposal for a Regulation of the European Parliament and of the Council Establishing a Social Climate Fund. COM (2021) 568 Final

EU (2021): Verordnung (EU) 2021/953 des Europäischen Parlaments und des Rates. https://eur-lex.europa.eu/legal-content/DE/ TXT/PDF/?uri=CELEX:32021R0953

Lerch, A./Rudolph, S.: Emissionshandel im Verkehrs- und Haushaltssektor? Ja, aber richtig! In: Ökologisches Wirtschaften 36/3: 12-13.

AUTOR + KONTAKT

Dr.Jan Nill ist Politikkoordinator in der Europäischen Kommission für die EU-Emissionshandelsreform, insbesondere die vorgeschlagene Emissionshandelserweiterung auf Gebäude und Verkehr.

Europäische Kommission, Generaldirektion Klimapolitik, Avenue de Beaulieu 31 1149 Auderghem (Brüssel) E-Mail: jan.nill@ec.europa.eu, Internet: https://ec.europa.eu/clima/policies/ eu-climate-action/delivering_de 